\title{
Experimental Observation of Continuous Melting into a Hexatic Phase
}

\author{
T. F. Rosenbaum, (a) S. E. Nagler, and P. M. Horn \\ IBM Thomas J. Watson Research Center, Yorktown Heights, New York 10598 \\ and \\ Roy Clarke \\ Department of Physics, The University of Michigan, Ann Arbor, Michigan 48109
}

(Received 6 April 1983)

\begin{abstract}
This paper reports the results of an x-ray diffuse scattering study of the melting transition of monolayer xenon on the surface of single crystals of exfoliated graphite. It is found that the two-dimensional xenon solid melts into an orientationally ordered liquid (or hexatic) phase. The temperature dependence of the orientational correlations suggests that the hexatic phase exists as a consequence of the continuous melting process, not the substrate.

PACS numbers: $64.70 . \mathrm{Dv}, 05.70 . \mathrm{Fh}, 64.60 .-\mathrm{i}$
\end{abstract}

In 1973 Kosterlitz and Thouless ${ }^{1}$ made the remarkable suggestion that melting of a two-dimensional solid could be continuous. Building on this prediction Nelson and Halperin ${ }^{2}$ and Young ${ }^{3}$ developed a comprehensive theory of the two-dimensional melting process. The most surprising and controversial aspect of this theory is the prediction that continuous melting is a two-step process with a bond-orientationally ordered liquid (or hexatic) phase separating solid from liquid. ${ }^{2}$ To date, bond-orientationally ordered liquids have been observed in intercalation compounds, ${ }^{4}$ adsorbates on surfaces, ${ }^{5}$ and liquid crystals. ${ }^{6}$ In each of these cases, however, one can argue that the orientational order exists independent of the melting process; in liquid crystals orientational order can be generated by the rodlike nature of the molecules while for adsorbates and intercalants it can be generated by substrate or host interactions. ${ }^{7}$ There exists, therefore, little experimental proof that the hexatic phase is a natural consequence of the continuous melting process.

In this Letter we report the results of an $x$-ray scattering study of the melting transition of monolayer xenon on single crystals of exfoliated graphite. A single-crystal substrate is necessary for the determination of bond-orientational correlations. In what follows we show that the two-dimensional xenon solid melts into a hexatic liquidcrystal phase. Furthermore, analysis of the data suggests that the hexatic phase exists as a consequence of the continuous melting process, not the substrate interaction.

In order to carry out this research project we were obliged to develop a new form of graphite, exfoliated single crystals. Briefly, single crystals of natural (New York State) graphite were carefully intercalated with $\mathrm{SbCl}_{5}$ to approximately stage eleven. These were then slowly heated in vacuum to $1100{ }^{\circ} \mathrm{C}$ between ground quartz plates. A solenoid was used to vary the compressional force on the graphite surface. The sample size was monitored optically and the pressure and temperature were varied concomitantly. Details of this procedure will be published elsewhere. ${ }^{8}$ Our best sample to date has an internal surface area of 6 Torr $-\mathrm{cm}^{3}$ and an in-plane mosaic of $2.3^{\circ}$ half width at half maximum. We anticipate that substantial improvements on these figures can be made. The results reported here are on our first sample which has 2 Torr $-\mathrm{cm}^{3}$ internal surface area and $\sim 3^{\circ}$ mosaic. The average step-free surface size was measured to be at least $4000 \AA$. This was determined by a lineshape analysis of a high-resolution $\mathrm{x}$-ray Bragg peak from the $\sqrt{3} \times \sqrt{3} R 30^{\circ}$ phase of adsorbed krypton. ${ }^{8}$

The xenon melting experiments were carried out on a two-axis $\mathrm{x}$-ray spectrometer with the use of $\mathrm{Cu} K a$ radiation from a Rigaku $12-\mathrm{kW}$ rotating-anode source. In-plane collimation was obtained with a vertically bent pyrolytic-graphite monochromator before and Soller slits after the sample. The xenon gas-handling system and cryostat were as discussed previously. ${ }^{9}$ The sample $\left(\sim 6 \times 6 \times 7 \mathrm{~mm}^{3}\right)$ was mounted with the $c$ axis vertical in an evacuable cell. The melting experiment was carried out with the sample cell open to a reservoir of xenon of approximately $200 \mathrm{~cm}^{3}$. The melting temperature and pressure, $140 \mathrm{~K}$ and 10 Torr, respectively, were chosen in a region of the phase diagram where the melting has been shown to be continuous. ${ }^{9,10}$ The experiments were carried out as a function of tempera- 
ture and the $\mathrm{x}$-ray intensity and pressure were monitored. Some deterioration of the sample was noted after multiple adsorptions and temperature cycling. ${ }^{8}$

Typical experimental results are shown in Fig. 1. An empty-cell background, corrected for xenon absorption, has been subtracted. The figure displays radial and angular scans through one of the six xenon $(1,0)$ hexatic spots. It is well known that at low temperatures the incommensurate xenon solid is rotated $30^{\circ}$ with respect to the graphite substrate. ${ }^{11}$ Within our uncertainties our high-temperature data agree with these findings. The solid lines through the data represent Lorentzian line-shape fits. The observed scattering above background is calculated by performing a mosaic average of the intrinsic line shape over the orientations of the substrate crystallites and convoluting the resulting function with the instrumental resolution. These fits are essentially identical to those done for $Z Y X$ graphite substrates except that the powder average is replaced by an average over the in-plane mosaic.,12 For radial scans there are five fitting parameters: intensity, Lorentzian width, peak position, substrate vertical mosaic, and xenon absorption correction. These parameters were determined by fits to the low-temperature data and the latter two were held constant. The $\omega$ scans involve three parameters: intensity, position, and Lorentzian width. The in-plane graphite mosaic and the instrumental resolution were measured and held constant. The fitted vertical

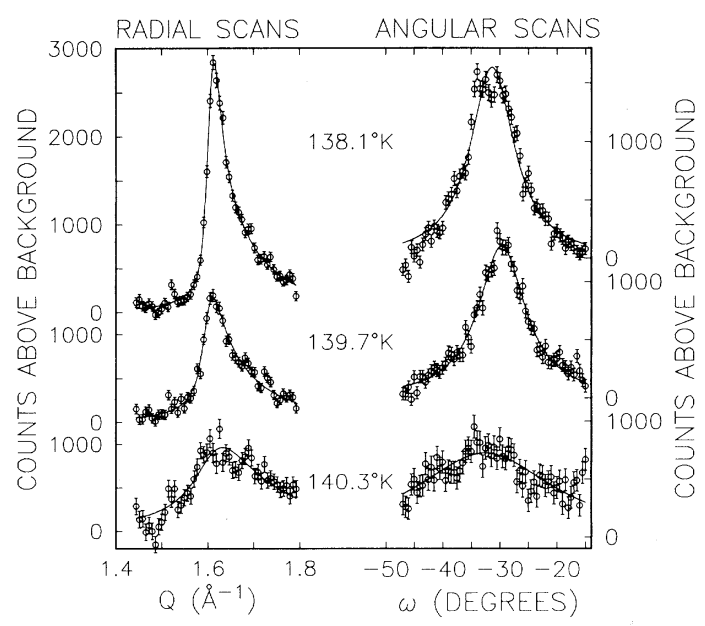

FIG. 1. Radial and $\omega$ scans through one of the six hexatic peaks. Typical scan times correspond to 15 min per point. mosaic was comparable to those found in $Z Y X$ graphite substrates. The line-shape fits are obviously satisfactory. Given that the observed scattering originates from many graphite surfaces, the existence of in-plane spots implies that the substrate-generated hexatic field ${ }^{7} h_{6}$ is large enough to align the different xenon layers with respect to the underlying substrate. As we will discuss below, the angular width of these diffuse peaks is strongly temperature dependent and is substantially larger than the radial width. This implies that the scattering is intermediate between typical Lorentzian diffuse scattering and the cylindrically symmetric scattering of a twodimensional liquid.

The significance of these data is perhaps best seen within the following theoretical framework. Within continuum elastic theory, the harmonic Hamiltonian for the hexatic phase is given $b y^{2,13}$

$$
H=\left(K_{A} / 2\right) \int_{a}^{L}(\nabla \theta)^{2} d^{2} r+\frac{1}{2} \int_{a}^{L} h_{6} \theta^{2} d^{2} r,
$$

where $\theta(r)$ represents the angle of a local xenonxenon bond and $K_{A}$ is a Frank constant similar to that found in liquid crystals. Near melting, $K_{A}$ is proportional to the positional correlation length squared $\left(\xi^{2}\right)$ with the proportionality constant being ${ }^{2}$ the dislocation core energy, $E_{c}$. The mean square angular fluctuations $\left\langle\delta \theta^{2}\right\rangle$ can be trivially estimated to be

$$
\left\langle\delta \theta^{2}\right\rangle=\frac{k_{\mathrm{B}} T}{2 \pi} \int_{2 \pi / L}^{2 \pi / a} \frac{q d q}{K_{A} q^{2}+h_{6}}+\frac{k_{\mathrm{B}} T}{h_{6} L^{2}},
$$

where we have explicitly separated out the $q=0$ term $k_{\mathrm{B}} T / h_{6} L^{2}$. In the limit that the substrate field $h_{6}$ is infinite, we have $\left\langle\delta \theta^{2}\right\rangle=0$, and the inplane scattering corresponds to six Lorentzian spots with width equal to the inverse radial correlation length, $1 / \xi$. For finite $h_{6}$, the angular width of the spots is greater than the radial width by a value $\left\langle\delta \theta^{2}\right\rangle^{1 / 2}$. In the limit that $h_{6} \rightarrow 0$, the $q=0$ term guarantees that $\left\langle\delta \theta^{2}\right\rangle \rightarrow \infty$ for any $L$ and the hexatic scattering should be an in-plane ring. ${ }^{14}$ However, finite $h_{6}$ cuts off this divergence and restores the hexatic spots. The condition that the $q=0$ term is negligible is just the condition required to align each xenon layer with respect to the substrate. For our system, this condition is trivially satisfied. When the substrate field is small but not zero, $(2 \pi / L)^{2} K_{A}$ $\gg h_{6} \gg k_{\mathrm{B}} T / L^{2}$, then $\left\langle\delta \theta^{2}\right\rangle=7 k_{\mathrm{B}} T / 2 \pi K_{A}$ where for our system we have approximated $\ln (L / a)=7$. Near melting, therefore, the excess angular width of the spot $\left\langle\delta \theta^{2}\right\rangle^{1 / 2}$ is proportional to the radial width $1 / \xi$. Nelson and Halperin predict that at 
sufficiently high temperatures, the hexatic phase will become unstable to the formation of free disclinations. $^{2}$ At this transition into the isotropic liquid phase, $K_{A}$ jumps discontinuously to zero from a universal value $K_{A}=72 k_{\mathrm{B}} T_{c} / \pi$. For our system, this corresponds to an orientational width $\left\langle\delta \theta^{2}\right\rangle^{1 / 2}=0.22 \mathrm{rad}=12.6^{\circ}$ or $|\overrightarrow{\mathrm{G}}|\left\langle\delta \theta^{2}\right\rangle^{1 / 2}$ $=0.35 \AA^{-1}$ (where $\overrightarrow{\mathrm{G}}$ is a reciprocal-lattice vector). Thus, the angular width of a hexatic spot should grow continuously until it reaches $0.35 \AA^{-1}$ at which point the spots should suddenly merge to form a liquid ring. Finite, nonzero $h_{6}$ will, of course, smear the transition and prevent the spot width from approaching infinity. The theoretical predictions for various values of $h_{6}$ are schematically shown in Fig. 2 where we plot the excess orientational width $\kappa_{\theta}=|\overrightarrow{\mathrm{G}}|\left\langle\delta \theta^{2}\right\rangle^{1 / 2}$ versus the radial width $\kappa_{r}=1 / \xi$.

In Fig. 3 we plot our experimental results for $\kappa_{\theta}$ vs $\kappa_{r}$. The solid and open circles represent different experimental runs through the melting transition. The agreement of the data with the small- $h_{6}$ limit of the theory is quite striking. Near melting $\kappa_{r}$ varies approximately linearly with $\kappa_{\theta}$. If we assume $K_{A}=2 E_{c}(\xi / a)^{2}$, we obtain $E_{c} \cong 3 k_{\mathrm{B}} T_{c} \cdot{ }^{2}$ The last datum point corresponds to a temperature which is approximately $1.2 \mathrm{~K}$ above the melting point. Above this temperature, the xenon scattering quickly becomes too broad and weak to resolve over background. It is tempting to interpret this strong temperature dependence of $\kappa_{\theta}$ as evidence for the approach of hexatic melting. This then suggests that the substrate-

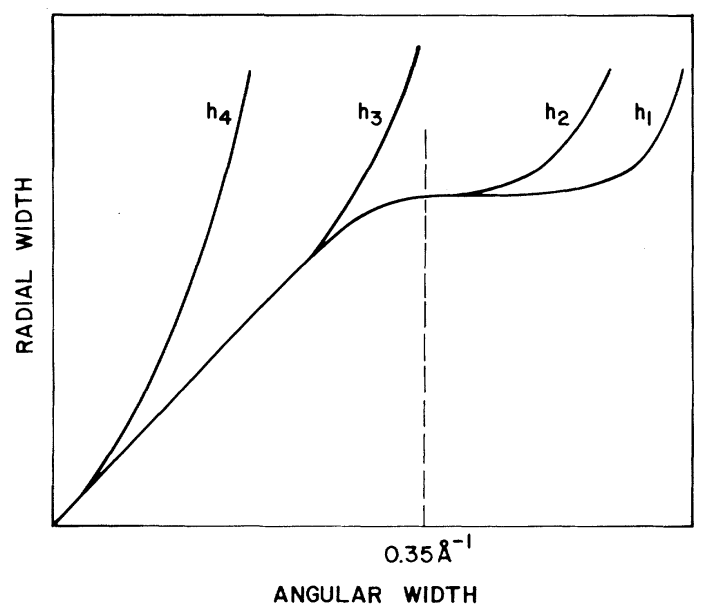

FIG. 2. Schematic of the heuristic dependence of the radial spot width $\kappa_{r}$ on the excess angular spot width $\kappa_{\theta}$ for various values of the substrate field $h_{6} . h_{4}>h_{3}$ $>h_{2}>h_{1}$. free hexatic melting temperature is at least 1.2 $\mathrm{K}(\sim 0.9 \%)$ above the melting temperature. $\mathrm{Al}-$ ternatively, this implies the existence of a substrate-free hexatic phase until the fluid correlation length $\xi$ decreases below $16 \AA$ ! The size of the hexatic region will presumably depend on the experimental parameters, especially the surface density. Perhaps most surprising is the apparent agreement of these results with the disclinationunbinding model of hexatic melting. ${ }^{2}$ Our data suggest that the hexatic melting transition occurs when $\kappa_{\theta}$ has a value in the vicinity of approximately $0.3 \AA^{-1}$. The agreement of this number with the predicted value of $0.35 \AA^{-1}$ is probably fortuitously good. Reasonable estimates ${ }^{7}$ for the magnitude of $h_{6}$ suggest that the substrate should start to become relevant when $K_{A}$ approaches its universal jump value $K_{A}=72 k_{\mathrm{B}} T_{c} / \pi$. Extension of these data to higher temperatures is certainly called for. Unfortunately, this must await the existence of better substrates and/or synchrotronbased experiments.

For the sake of completeness, we have tried to fit our data with other models of the melting transition. Specifically, one can try to describe the data as an isotropic liquid with in-plane order being generated solely by $h_{6}$. To obtain the observed $\left\langle\delta \theta^{2}\right\rangle$ with even a highly correlated liquid requires $h_{6} \gtrsim 10 k_{\mathrm{B}} T$ (approximately three orders

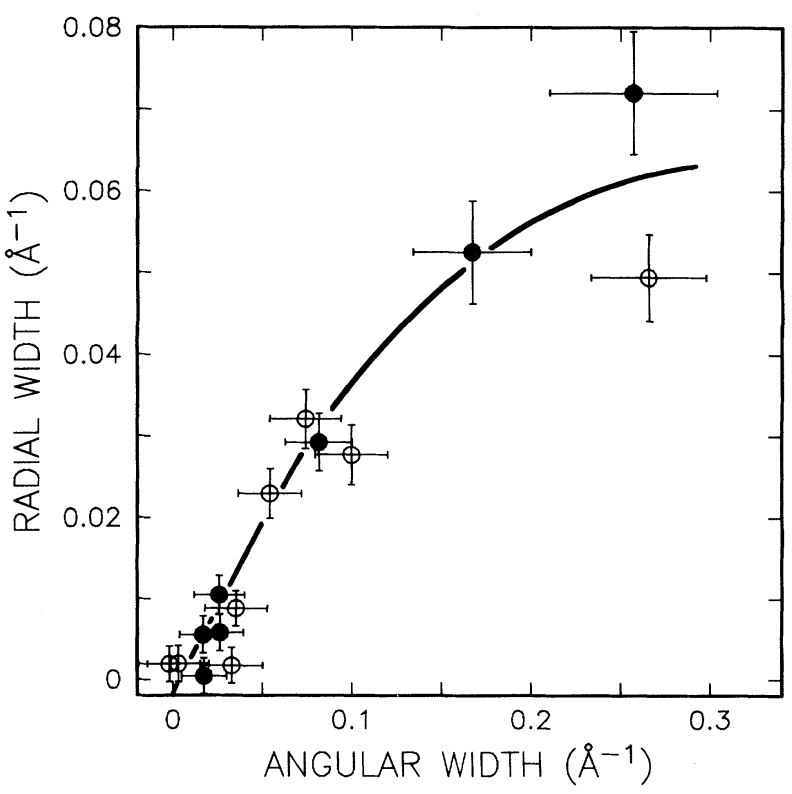

FIG. 3. Experimental results for the dependence of the radial spot width $\kappa_{r}$ on the excess angular spot width $\kappa_{\theta}$. The solid line is a guide to the eye. 
of magnitude greater than its estimated value ${ }^{7}$ ). Furthermore, this scenario cannot reproduce the observed temperature dependence of the angular width. Similarly, our observed line shapes rule out liquid-solid coexistence as the origin of the orientational order. This, of course, does not imply that some other, more clever alternative explanation cannot be found.

In conclusion, our data suggest that the hexatic phase is indeed a consequence of the continuous melting process. We have argued that the substrate determines the average orientation of the xenon layers but does not create the hexatic order within each platelet. The existence of a substratefree hexatic phase provides a dramatic confirmation of the dislocation-unbinding mechanism of melting suggested by Kosterlitz and Thouless, ${ }^{1}$ Halperin and Nelson, ${ }^{2}$ and Young. ${ }^{3}$ Furthermore, the data are in qualitative agreement with the disclination-unbinding model for the melting of the hexatic phase. ${ }^{2}$ Finally, from a more global perspective, the existence of high-quality exfoliated single-crystal substrates should have a substantial impact on studies of other surface phase transitions.

It is a pleasure to thank R. J. Birgeneau, P. Dimon, D. R. Nelson, and D. M. Sutton for numerous helpful discussions and H. Homma for help with sample preparation. Special thanks are due to G. Grinstein for numerous corrections, discussions, and helpful comments. One of us (S.E.N.) was supported by a Natural Sciences and Engineering Research Council of Canada Postdoctoral Fellowship and the work was supported in part by the National Science Foundation Low Temperature Physics Grant No. DMR-8207449.

(a) Present address: The James Franck Institute, University of Chicago, 5640 Ellis Avenue, Chicago, Ill. 60637.

${ }^{1}$ J. M. Kosterlitz and D. J. Thouless, J. Phys. C $\underline{6}$, 1181 (1973).

${ }^{2}$ B. I. Halperin and D. R. Nelson, Phys. Rev. Lett. 41, 121 (1978); D. R. Nelson and B. I. Halperin, Phys. Rev. B 19, 2456 (1979).

${ }^{3}$ A. P. Young, Phys. Rev. B 19, 1855 (1979).

${ }^{4}$ Roy Clarke, N. Caswell, S. A. Solin, and P. M. Horn, Phys. Rev. Lett. 43, 2109 (1979).

${ }^{5}$ C. G. Shaw and S. C. Fain, Surf. Sci. 83, 1 (1979).

${ }^{6}$ R. Pindak, D. E. Moncton, S. C. Davey, and J. W. Goodby, Phys. Rev. Lett. 46, 1135 (1981).

${ }^{7}$ J. P. Mc Tague and A. D. Novaco, Phys. Rev. B 19 , 5299 (1979).

${ }^{8}$ Roy Clarke, P. M. Horn, and T. F. Rosenbaum, to be published.

${ }^{9}$ P. A. Heiney, R. J. Birgeneau, G. S. Brown, P. M. Horn, D. E. Moncton, and P. W. Stephens, Phys. Rev. Lett. 48,104 (1982).

${ }^{10}$ P. Dimon, P. M. Horn, R. J. Birgeneau, M. Sutton, and D. E. Moncton, to be published.

${ }^{11}$ P. S. Schabes-Retchkiman and J. A. Venables, Surf. Sci. 105, 536 (1981).

${ }^{12}$ P. W. Stephens, P. A. Heiney, R. J. Birgeneau, P. M. Horn, D. E. Moncton, and G. S. Brown, to be published.

${ }^{13}$ We thank D. R. Nelson (private communication) for pointing out the importance of this Hamiltonian and of finite-size effects.

${ }^{14}$ In liquid crystals this divergence can be cut off by pinning of the orientational direction at the boundary. 